\title{
Evaluation of Efficacy of Omega 3 Fatty Acid Supplementation in Obese Patients: A Pilot Study
}

\author{
Dr. Suramya S. ${ }^{1}$, Dr. Sheela Kumar Gujjari ${ }^{2}$, Dr. Nada Ali ${ }^{3}$ \\ ${ }^{I}$ (Department of Periodontology, JSS Dental College and Hospital, JSS University, India) \\ ${ }^{2}$ (Department of Periodontology, JSS Dental College and Hospital, JSS University, India) \\ 3 (Department of Periodontology, JSS Dental College and Hospital, JSS University, India)
}

\begin{abstract}
This was a pilot study with 30 patients, including both males and females to evaluate the efficacy of omega 3 fatty acid capsules in obese patients with periodontal disease. The subjects were divided into test and control groups. Only the test group patients received omega 3 fatty acid supplementation (2g/day) daily over a period of three months. Scaling and root planing was performed as part of periodontal therapy for all the patients. The parameters assessed were total lipid profile, CRP and periodontal status. At the end of the study, there was a positive impact seen in both the control and test groups in all the clinical parameters. Total cholesterol reduced significantly more in the test group than in the control group. An identical trend was observed in the LDL and Triglyceride levels. HDL levels were increased across the groups. CRP was reduced significantly for the test and control groups were compared, the mean difference was insignificant. Based on these primordial results it was concluded that omega 3 fatty acid supplementation can be used as an adjuvant in treatment of obese patients with periodontal disease.
\end{abstract}

Keywords: Lipid profile, obese patients, Omega 3 fatty acid capsules, periodontal status, scaling and root planing.

\section{Introduction}

In the contemporary world, obesity is one of the many demons affecting mankind. A global epidemic today, this chronic systemic disease is prevalent in both developing and developed countries affecting both children and adults. Obesity can be broadly defined as the body mass index $>30.0 \mathrm{~kg} / \mathrm{m}^{2}$ and is a major public health problem. It predisposes an individual to a variety of co-morbidities and complications that affect overall health. The worldwide prevalence of obesity is a considerable source of concern given its potential on morbidity, mortality and the cost of healthcare. WHO recognizes obesity as a predisposing factor to major chronic diseases ranging from cardiovascular disease to cancer [1].

Cross-sectional studies suggest that obesity is associated with oral diseases, particularly periodontal disease. Prospective studies also suggest that periodontitis itself may be related to cardiovascular disease [2]. The possible causal relationship between obesity and periodontitis and the potential underlying biological mechanisms yet remains to be established. However, it is known that the adipose tissue does actively secretes a variety of cytokines and hormones that are involved in inflammatory processes, pointing toward similar pathways involved in the pathophysiology of obesity, periodontitis, and related inflammatory diseases [2].Thus, obese patients with a high serum triglyceride level and / or low HDL - cholesterol could be at higher risk of periodontal infection [3].

Advances in understanding the etiology and pathogenesis of periodontitis have led to increasingly effective pharmacological interventions. The use of antibiotics has drawbacks like systemic adverse effects and increased incidence of microbial resistance. Besides antibiotics, there has been a plethora of adjuvants along with the conventional periodontal therapy. These include products of plant and animal origin.

n-3 polyunsaturated fatty acids (PUFAs) are long chain PUFAs found in plants and marine sources such as fish, mussel, oyster, shrimp but primarily cold water fish but also exist in a wide range of plant products such as nuts, seeds and vegetable oils such as soybean[4].The essential roles of omega-3 PUFAs in health were evident since1929. Omega-3 PUFAs are widely held to act via several possible mechanisms, such as preventing conversion of arachidonate to proinflammatory eicosanoids or serving as an alternative substrate producing lesspotent products [5-6]. These omega 3-polyunsaturated fatty acids, including docosahexanoic acid and eicosapentanoic acid have shown to have therapeutic value and anti-inflammatory and protective value in diseases like rheumatoid arthtitis, asthma, atherosclerosis ,cancer, cardiovascular diseases as well as periodontitis[7].

A recent study concluded that therapeutic omega-3 fatty acids significantly reduced the gingival tissue levels of PGE2, PGF2 $\alpha$, LTB4, and PAF in experimental periodontitis, suggesting that its therapeutic usage may have beneficial effects in treating periodontitis. Furthermore, prophylactic usage of omega-3 provided additional beneficial effects by decreasing the gingival tissue level of these mediators to levels of the healthy tissue [8]. 
Recently, human studies have also found growing body of evidence indicating that increasing the intake of n-3 PUFA by $0.3-3.0 \mathrm{~g}$ /day can reduce body weight and body fat in overweight and obese individuals[9]. This can be attributed to the fact that fatty acids are responsible for many functions of immune cells such as regulation of gene expression by either altering receptor activity, signaling or activation of transcription factors, and the production of lipid mediators such as prostaglandins and leukotrienes. Therefore, alteration of the fatty acid composition in the cell membrane may result in changes of the types of lipid mediators synthesized and the subsequent inflammatory cascade.

With the mentioned background, the present study was conducted to correlate the dynamics of the effect of omega 3 fatty acid supplementation in obese patients with periodontitis. The clinical and lipid parameters were used to estimate the changes in the periodontal status and lipid profile.

\section{Materials And Methods}

This study was conducted in the Department of Periodontology, JSS Dental College and Hospital, Mysore, an affiliated institution of the JSS University, Mysore. This was a single blinded comparative study conducted over a period of 3 months.

\subsection{Ethical clearance and informed consent}

The nature and design of the clinical trial was explained to the patients. A prior written informed consent was taken from all the participants of the study. The investigation was performed in accordance to the requirements of the "Declaration of Helsinki." The study protocol explained herein was approved by the Institutional Review Board (IRB), JSS Dental College and Hospital, an affiliated institution of the JSS university, Mysore.

\subsection{Patient selection}

The patients participating in this study were selected from the outpatients visiting the Department of Periodontology, JSS Dental College and Hospital, Mysore, a constituent college of JSS university. Subjects included 40 obese individuals (BMI $>30$ ), above 30years of age were selected. They were randomly assigned to either group A: test $(n=20)$ or Group B: control $(n=20)$ by computer generated random tables.

\subsection{Inclusion Criteria \& Exclusion Criteria:}

Obese patients (BMI $>30 \mathrm{Kg} / \mathrm{m}^{2}$ ) with Chronic generalised Periodontitis, more than 30 yrs in age, with at least 20 natural teeth and taking a mixed diet were included in the study. Smokers, patients who received periodontal surgery / antibiotic therapy in the past 3 months, patients on alternative nutrition supplements, pregnant / nursing women, evidence of localized aggressive periodontitis / necrotizing ulcerative gingivitis, patients Allergic to fish / sea food and Patients on anti cholesterol drugs were excluded from the study.

\subsection{Clinical parameters:}

The clinical parameters were measured at baseline, 1 month and 3 months including plaque Index, gingival Index and sulcus bleeding index. Probing pocket depth and clinical attachment level was measured at six points around the tooth surface with UNC 15 probe. Kappa analysis was done to ensure intra- examiner reliability before the clinical parameters were measured (Kappa coefficient $=0.85)$.

\subsection{Total lipid profile and $\mathrm{C}$ - reactive protein}

Laboratory evaluation of the lipid status was done for each patient. The patients were sent to various laboratories in and around Mysore and serum samples were collected. LDL lipoprotein, HDL lipoprotein, total cholesterol and triglycerides were estimated. C - Reactive protein was also measured. The samples were obtained after a 12 hour fasting period. The measurements were taken at baseline, 1 month and 3 months. The body mass index was calculated for each patient at baseline, 1 month and 3 month. This was not used as part of statistical analysis.

\subsection{Study Design and Intervention:}

After informed consent was obtained, detailed histories were recorded to assess any allergies to fish or seafood, family and personal medical history of cardiac disease and risk factors, and dietary intake of fish and seafood. All selected patients were randomized into two groups such that group A (Test group) who received full mouth scaling and root planing along with omega -3 fatty acid supplements and group B (Control group) who received full mouth scaling and root planing only.

This study was designed to determine the effect of $2000 \mathrm{mg} /$ day of mixed EPA/DHA omega-3 fatty acid supplements (550 mg EPA; $450 \mathrm{mg}$ DHA) per 2 soft gels of Seven seas on obese individuals with chronic generalized Periodontitis. So, the 20 individuals that were randomized to the test group were provided individual 
bottles of 14 fish oil soft gels and instructed to take 2 on a daily basis. These bottles were collected on a weekly basis to access compliance and new bottles were provided. The control subjects did not receive any additional supplements and were asked not to take any essential fatty acid supplements. Compliance and side effects data were collected using weekly questionnaires. Patients were recalled and serum lipid profiles, CRP levels and periodontal clinical parameters were assessed at baseline, 1 month and 3 months.

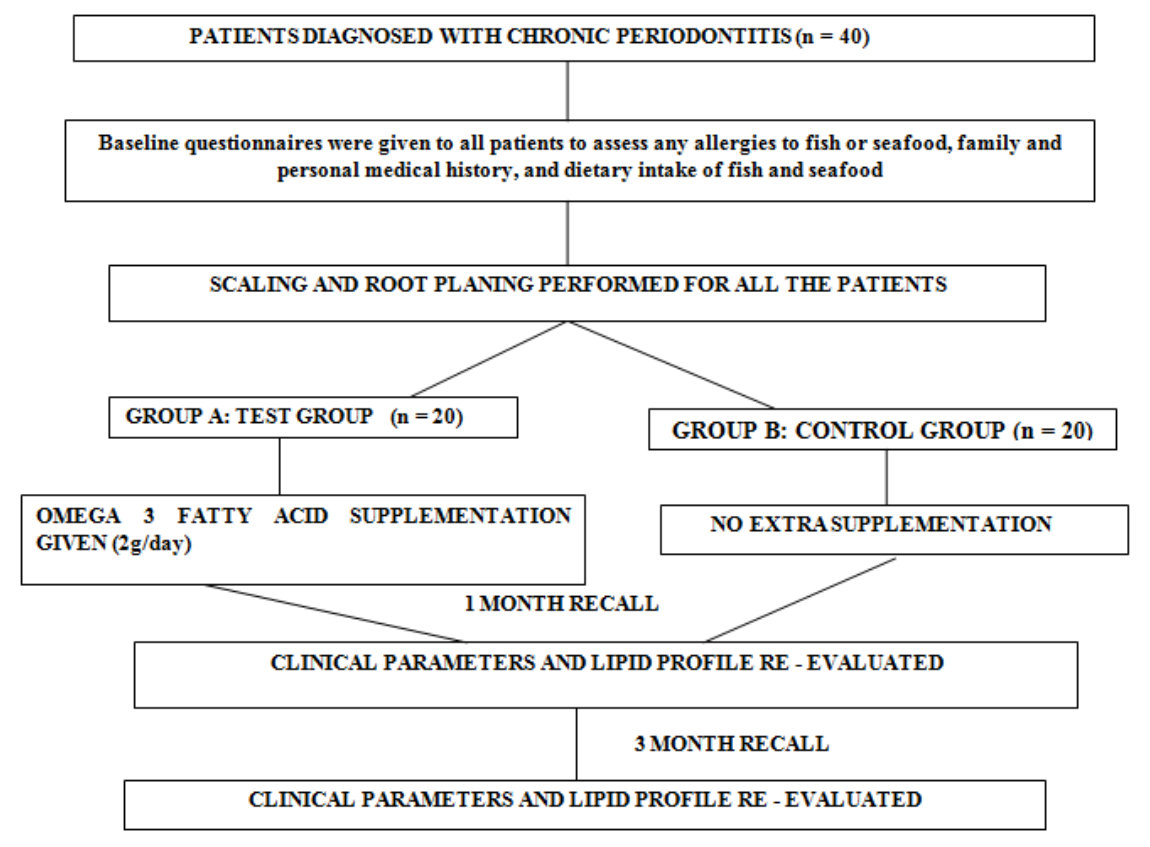

\section{Statistical Analysis}

The obtained data was subjected to statistical analysis. Descriptive statistics was used to describe the demographic data. One way ANOVA and Repeated measure ANOVA was used for multivariate analysis. Paired samples " $\mathrm{t}$ " test was used evaluate the changes in all the parameters over the study period.

\section{Results}

The present study was conducted to assess the efficacy of omega fatty acid capsules in obese patients with periodontal disease. The parameters assessed were total lipid profile (HDL, LDL, Cholesterol, and Triglycerides), CRP and periodontal status. The study population included both males and females. Figure 1 displays the demographics of the test and control groups.

There was a positive impact seen in both the groups in all the clinical parameters at the end of the study. There was a statistically significant reduction $(\mathrm{p}<0.05)$ in the plaque scores, gingival scores and bleeding on probing in the test groups at 1 month and they remained significant low at the 3 month interval. Similar trends were observed in the control group (Figure 2-4, Table 1-3). When the test and the control were compared for these parameters the intergroup comparison showed a non significant difference $(p>0.05)$.

At baseline the mean probing depths were not significantly different between test and control groups ( $\mathrm{p}>0.05$ ). The intervention of omega 3 fatty acid resulted in mean probing pocket depth reduction of $4.30 \mathrm{~mm}$ and $4.56 \mathrm{~mm}$ in the control and test groups respectively at the end of 3 months (Figure 5, Table 4). On intergroup comparison, the difference was insignificant ( $p>0.05)$. When clinical attachment levels were measured, there was also significant gain seen in both the groups. There was a gain attachment of $1.15 \mathrm{~mm}$ and $0.94 \mathrm{~mm}$ in the test and control groups over the entire period of the study (Figure 6, Table 5) $(\mathrm{p}<0.05)$.

Besides the clinical parameters, the lipid status of all the subjects was also evaluated. The lipid profile parameters showed a variable trend. Total cholesterol reduced significantly more in the test group by a value of $6.2 \mathrm{mg} / \mathrm{dl}$ compared to $1.2 \mathrm{mg} / \mathrm{dl}$ in the control group over the 3 month time period (Figure 7, Table 6). Compared to the test and control groups the difference remained statistically significant. The HDL levels were increased as the lipid status improved. HDL levels elevated from $38.2 \mathrm{mg} / \mathrm{dl}$ to $41.7 \mathrm{mg} / \mathrm{dl}$ for the test group and $36.8 \mathrm{mg} / \mathrm{dl}$ to $38.3 \mathrm{mg} / \mathrm{dl}$ for the control group (Figure 8, Table 7). The LDL levels reduced significantly for test group and control group (Figure 9, Table 8). The reduction was significantly higher for the test group compared to the control. The triglyceride levels were significantly reduced from $221.9 \mathrm{mg} / \mathrm{dl}$ to $214.8 \mathrm{mg} / \mathrm{dl}$ for the test group. For the control group the levels were slightly elevated at the end of the study. This change was insignificant (Figure 10, Table 9). 
CRP at baseline for the test group was 9.74 which decreased to 7.47 by the end of 3 months. In the control group this change was seen from 9.73 to 8.20 . Both these reductions were statistically significant (Figure 11, Table 10). When the test and control groups were compared, the mean difference was insignificant. When the clinical parameters were correlated to the alteration in the lipid profile, positive change was observed.

\section{Discussion}

With the increased understanding of periodontal disease it is now accepted that although the primary etiology of periodontal disease in bacterial, but it is the host inflammatory response that plays a critical determinant in the pathogenesis of periodontitis. The failure of resolution of inflammation leads to the overproduction of inflammatory response. New exciting treatment of periodontitis aims at the resolution of inflammation which is the latest paradigm in the same field. Omega 3 fatty acids are known to have therapeutic value, anti inflammatory and protective actions in the various chronic diseases including periodontitis. The beneficial action of w-3 fatty PUFA's is through the reduction in the classic inflammatory mediators such as prostaglandins and through the enzymatic conversion to novel series of lipid mediators named resolvins and protectins. Few studies conducted in both animal and human models have reinforced these facts. Along with the effect on inflammatory periodontal disease, data also suggest the role of omega 3 fatty acid on improving the lipid profile in obese patients. With the background, the present study was aimed at improving the periodontal and lipid status in obese patients. The results were encouraging as the patients receiving daily supplementation of omega 3 fatty acid capsules showed marked improvement.

The subjects in the present study were obese (BMI $>30 \mathrm{Kg} / \mathrm{m}^{2}$ ) and suffered from periodontal disease. The link between obesity and periodontitis has been an area of research in the past. Data suggest that obese patients stand at a higher risk of diseased periodontium, suggesting it as a risk factor. It has been suggested that obesity is second only to smoking as the strongest risk factor for inflammatory periodontal tissue destruction [10]. The first report on the relationship between obesity and periodontal disease appeared in 1977, when Perlstein et al. observed histopathologic changes in the periodontium in hereditary obese Zucker rats. Later on, the hypothesis of obesity as a risk factor for periodontal disease was supported by epidemiological studies. The analysis of National Health and Nutrition Examination Survey (NHANES III) data and demonstrated that BMI was positively correlated with the severity of periodontal attachment loss; they found that this relationship is modulated by insulin resistance [11].

Hyperlipideamia often co-exists with obesity and is one of possible mechanism explaining the association between obesity and periodontal disease[12]. Hyperlipidaemia is a state of abnormal lipid profile, which is characterized by elevated blood concentrations of triglycerides, elevated level of total cholesterol and low density lipoprotein- cholesterol(LDL), and decreased level of high- density lipoprotein- cholesterol (HDL) [12]. A recent study suggests that periodontal infection has a definite role in altering lipid metabolism leading to hyperlipidemia [13]. In a large cohort study, the presence of periodontal pockets as measured by CPITN was positively correlated with total cholesterol and LDL - cholesterol [14]. In another study conducted on Japanese adults, it was found that high upper body obesity and high total body fat were correlated with higher risk of periodontal disease, compared with normal weight persons [15] An Indian study also concluded that good oral hygiene and normal body weight can reduce the overall inflammatory burden, thereby reducing the risk for development of periodontal disease. In our study also patients were given regular oral hygiene instruction and basic periodontal therapy. There was a considerable improvement seen in their oral and systemic health [16].

Fish oil is a main source of omega-3 fatty acids where the major polyunsaturated fatty acid components are eicosapentaenoic acid (EPA) and docohexaenoic acid (DHA). EPA and DHA compete with AA for the COX and LOX pathways, which result in the reduced synthesis of highly active AA metabolites[17]. Thus, omega-3 fatty acids show anti-inflammatory, anti-thrombotic, anti-arrhythmic, hypolipidemic, and vasodilator effects. With these properties they are indicated in the treatment of some chronic inflammatory diseases such as rheumatoid arthritis and psoriasis [18-19]. In the same light recent studies have evaluated the beneficial effects of fish oil as a rich dietary sources of omega -3 poly unsaturated fatty acids on diverse physiological processes in the body and on a variety of chronic inflammatory diseases, including periodontal diseases [20]. The efficacy of omega 3 fatty acids has been tested in invitro and invivo studies.

Vardar et al. evaluated the use of omega-3 fatty acids with the purpose of blocking the arachidonic acid cascade in endotoxin-induced periodontal disease in rats. Experimental periodontitis was induced by repeated injections of Escherichia coli lipopolysaccharide (LPS). Therapeutic omega-3 fatty acids significantly reduced the gingival tissue levels of PGE2, PGF2-alpha, LTB4, and PAF in experimental periodontitis. Furthermore, the prophylactic usage of omega-3 fatty acids provided additional beneficial effects, by decreasing the levels of these mediators in the gingival tissue to the levels found in healthy tissues [21]. Sun et al. studied the EPA, DHA, and PUFA present in fish oil and showed that they decreased osteoclast activation in vitro [22]. A study by Kesavalu et al. showed that P. gingivalis-infected rats treated with omega-3 fatty acids had significantly less alveolar bone resorption. The supplement diet was effective in modulating alveolar bone resorption following P. 
gingivalis infection, and could be a useful adjunct in the treatment of periodontal disease [23]. Hasturk et al. showed topical application of bioactive products derived from omega-3 fatty acids, including DHA and EPA, conferred dramatic protection against inflammation-induced tissue and bone loss associated with periodontitis in the experimental models [24].

A double-blind, randomized pilot study was conducted by Campan et al. to evaluate the antiinflammatory action of omega-3 fatty acids on experimentally-induced gingivitis in humans. They demonstrated that n-3 PUFA induced a tendency toward reduced inflammation, but were unable to conclude significant efficacy [25]. Eberhard et al. studied the clinical effects of the topical application of omega- 6 and n-6 polyunsaturated fatty acids in patients with experimental gingivitis. Rinsing with omega- 6 fatty acids could reduce the level of GCF in established experimental gingivitis [26]. Rosenstein et al. examined the potential anti-inflammatory effects of PUFA supplementation, by administration of fish oil as a source of the omega-3 PUFA and EPA, and borage oil as a source of GLA, to adults with periodontitis. Improvement in gingival inflammation was observed in subjects treated with borage oil, with the trend apparent in subjects treated with fish oil or a combination of PUFA. Improvement in probing depth was seen in those subjects treated with either fish oil alone or borage oil alone, but a statistical significance was only seen after comparison of borage oil and placebo $(\mathrm{P}<0.044)$. The use of borage oil supplementation could have beneficial effects on periodontal inflammation. Omega-6 PUFA supplementation seemed to offer more impressive results than either omega-3 PUFA supplementation or the combination of lower doses of both supplements [27]. In a nationally representative cross-sectional study of 9182 adults, aged 20 years, by Naqvi et al., higher dietary intake of omega-3 fatty acids, particularly DHA and EPA, were inversely associated with the prevalence of periodontitis. The results also suggested that DHA could be more potent in influencing periodontitis[28]. El-Sharkawy et al. studied the daily dietary supplementation with omega-3 fatty acids and low-dose aspirin in a double-masked clinical study. They found that supplementation with omega-3 with aspirin resulted in a significant shift in the frequency of pockets, with probing depths of less than $4 \mathrm{~mm}$ [29]. Additional daily supplementation of omega-3 fatty acids showed a reduction in periodontal disease and gingival pocket formation, with an increase of attachment in periodontal disease patients, indicating that omega-3 fatty acids are protective against inflammatory bone loss.

The above mentioned studies display the positive impact of omega 3 fatty acid on periodontitis. The present study is in line with these researches.

Animal studies have shown that incorporating n-3 PUFA into high-fat obesogenic diets fed to rodents reduces body fat accumulation [31,32] although one study in diabetic rats reported that supplementing a high-fat diet with LC n-3 PUFA exacerbated, rather than reduced, weight gain[33].

Calabresi et al [34] evaluated patients who received 4 capsules daily of Omacor (providing $3.4 \mathrm{~g}$ EPA and DHA per day) or placebo for 8 weeks in a randomized, double-blind, crossover study. They found that Omacor significantly lowered plasma triglycerides and vLDL-C levels by $27 \%$ and $18 \%$, respectively. Piolot et al[35] reported significant reductions in vLDL triglyceride and vLDL-C concentrations following daily ingestion of 6 grams of omega-3 EFA (EPA/DHA) during a 2-month period $(P<.05)$. Rivellese et al evaluated the effects of omega-3 EFA on lipid metabolism in healthy individuals undergoing either a diet rich in monounsaturated fats or one rich in saturated fatty acids [36]. In a recent study by Yates et al conducted a 2 month study to evaluate the lipid profile of profile of professional football players on omega 3 fatty acid supplementation. Treatment increased high-density lipoprotein, decreased triglycerides treatment, very lowdensity lipoprotein treatment, intermediate density lipoprotein, remnant lipoproteins and very low-density lipoprotein-3. An average increase of $106.67 \%$ for docosahexaenoic acid and $365.82 \%$ for eicosapentaenoic acid compared to control was also shown [37].

Our study showed similar results with the improvement seen on the lipid status when the HDL increased, and there was a reduction in the triglyceride, LDL and total cholesterol. In contrast EPA and DHA levels as well as the estimation of $v$ LDL was not done.

One of the few contrasting study to the present one is the one conducted by Goh YK et al to Effect of omega 3 fatty acid on plasma lipids, cholesterol and lipoprotein fatty acid content in NIDDM patients. LDL and HDL cholesterol, insulin, glucagon and C-peptide levels were not affected by either omega 3 supplement. It is concluded that a modest intake of omega 3 fatty acids, such as could be obtained from consuming fish regularly, will reduce plasma triglyceride level without affecting LDL or HDL cholesterol levels [38]. The results may be attributed to the diabetic status of the subjects. All patients in our study were non diabetic.

In a systematic review and meta-analysis on CRP in relation to periodontitis, Paraskevas et al. [39] have concluded that the serum CRP level in patients with periodontitis is elevated compared with healthy individuals and that periodontal therapy results in the lowering of CRP levels. Hence, we used the serum CRP level as a biochemical parameter to evaluate the effect of $\omega-3$ PUFAs as an adjunctive therapy option used in conjunction with SRP. 
Vardar-Sengul et al. [40] demonstrated that both the prophylactic and therapeutic use of $\omega-3$ PUFAs in experimental periodontitis did not change the serum CRP level when compared to a placebo group in a rodent model. Mori et al. [41] reported that supplementation with purified EPA or DHA for 6 weeks in nonsmoking diabetic subjects did not lead to any significant change in the serum CRP level compared to placebo controls. Madsen et al. [42] administered $\omega-3$ PUFAs at two different doses (6.6 g/day or $2.0 \mathrm{~g} /$ day) to healthy volunteers for 12 weeks and found no change in serum CRP concentrations after either dose of $\omega-3$ PUFAs. Geelen et al. [43] evaluated the effects of $\omega-3$ PUFAs in a placebo-controlled, double-blind study with 84 middle-aged subjects. The subjects were given capsules of either $\omega-3$ PUFAs or a placebo for 12 weeks, and the results indicated no significant differences in serum CRP concentrations [43].

Like in afore mentioned studies, there was a minimal change in the CRP levels. Yet, in contrast to the same studies the CRP changes in our test group were statistical significant on analysis. The cause for this variance may be better patient compliance due to Hawthorne effect. The exact cause for this difference is requires further investigation.

Although given research was conducted with utmost planning and precision, it has its limitations. This includes the relatively small sample size and the limited duration of the study. BMI was measured as part of routine investigation, but the value was not considered to statistical analysis. This was done in order to make the results of the study uncomplicated and precise.

According the best of our knowledge the present research was one of the few where the use of omega 3 fatty acid supplementation has been used a mode to resolute periodontal inflammation and also improve the lipid profile. It also has an aspect where the systemic inflammatory status is targeted through the estimation of CRP levels. The uniqueness of this study is highlighted as it also forms a link between the oral cavity and human body. Although results seen were remarkable, there is a sincere need to conduct more long term researches and randomized clinical trial. Following the trend of our study, the supplementation of omega 3 fatty acid capsules can indeed be a revolution for the treatment of obese patients helping them improve their oral and systemic health.

\section{Conclusion}

The use dietary omega-3 fatty acid has shown great promise in field of periodontal therapy the as well as and its effect on obesity. The present study was conducted to harmonize the effect of this natural nutrient source on obese patients suffering from periodontitis. The results were positive with significant improvement seen in the test group patients. The intake of omega 3 fatty acid supplementation effectively reduced hyperlipedimia of these patients and also improved the periodontal status. Thus it this study can be considered as a small step in the ongoing revolution of host modulatory mechanisms to treat chronic diseases. Further long term researches must be done to further strengthen the obtained results.

\section{Acknowledgements}

This research was conducted by the financial grant provided by JSS University, Mysore. Authors sincerely acknowledge their support and encouragement.

\section{References}

[1]. Benjamin W.Chaffee et al. Association between chronic periodontal disease and obesity: a systematic review and meta analysis. Journal of Periodontology 2010; 81:1708-1724.

[2]. Obesity, Inflammation, and Periodontal Disease N. Pischon,N. Heng, J.-P. Bernimoulin, B.M. Kleber, S.N. Willich T. Pischon. JDR May 2007 vol. 86 no. 5 400-409

[3]. Saxlin et al. Association between serum lipid levels and periodontal infection. Journal of Clinical Periodontology 2008; 35: 10401047.

[4]. Friedman, A. \& Moe, S. (2006). Review of the effects of omega-3 supplementation in dialysis patients. Clinical Journal of American Society of Nephrology, 1, 182-192.

[5]. Serhan CN. A search for endogenous mechanisms of anti-inflammation uncovers novel chemical mediators: missing links to resolution. Histochem Cell Biol 2004; 122:305-21.

[6]. Arita M, Bianchini F, Aliberti J, Sher A, Chiang N, Serhan CN, et al. Stereochemical assignment, anti-inflammatory properties, and receptor for the omega-3 lipid mediator resolvin E1. J Exp Med 2005; 201:713-22

[7]. Heshman et al. Adjunctive treatment of chronic periodontitis with omega -3 fatty acids and low dose aspirin.journal of periodontology 2010;81:1635-1643

[8]. Saynur Vardar, Eralp Buduneli, Oya Türko־glu et al. Therapeutic Versus Prophylactic Plus Therapeutic Administration of Omega-3 Fatty Acid on Endotoxin-Induced Periodontitis in Rats. Journal of Periodontology 2004; 75:1640-1646.

[9]. D.Buckely et al. Long chain of omega - 3 fatty acids may be beneficial for reducing obesity - a review. Nutrients. 2010 December; 2(12): 1212-1230

[10]. Nishida N, Tanaka M, Hayashi N, Nagata H, Takeshita T, Nakayama K, et al. Determination of smoking and obesity as periodontitis risks using the classification and regression tree method. J Periodontol. 2005;76:923-8.

[11]. Genco RJ, Grossi SG, Ho A, Nishimura F, Murayama Y. A proposed model linking inflammation to obesity, diabetes, and periodontal infections. J Periodontol. 2005; 76:2075-84.

[12]. Saxlin et al. Association between serum lipid levels and periodontal infection. Journal of clinical periodontology 2008; 35 : 10401047 . 


\section{Evaluation of Efficacy of Omega 3 Fatty Acid Supplementation in Obese Patients: A Pilot Study}

[13]. Swati Penumarthy, Gautami S. Penmetsa, Satheesh Mannem. Assessment of serum levels of triglycerides, total cholesterol, high-density lipoprotein cholesterol, and low-density lipoprotein cholesterol in periodontitis patients. Journal of Indian Society of Periodontology - Vol 17, Issue 1, Jan-Feb 2013

[14]. Katz et al. Association between periodontal pockets and elevated cholesterol and low density lipoprotein cholesterol levels. Journal of periodontology 2002; 73:494-500

[15]. N.Pischon et al. Obesity, inflammation and periodontal disease. Journal of dental research 2007;86(5):400-409

[16]. Giri DK, Kundapur PP, Bhat GS, Bhat KM, Guddattu V. Periodontal disease and obesity in an Indian population .NJMS ; Volume 02 (Number 02 ) : July-December 2013

[17]. Van der Merwe CF. A different and physiological approach to manipulating the inflammatory response. Eur J Gastroenterol Hepatol 1993;5:433-436.

[18]. Ariza-Ariza R, Mestanza-Peralta M, Cardiel MH. Omega-3 fatty acids in rheumatoid arthritis: An overview. Semin Arthritis Rheum 1998; 27:366-370.

[19]. Bittiner SB, Cartwright I, Tucker WFG, Blehen SS. A double-blind, randomized, placebo-controlled trial of fish in psoriasis. Lancet $1988 ; 3: 378-380$.

[20]. Fernandez G, Venkatraman J. Role of omega -3 fatty acids on health and disease. Nutri Res 1993: 13: S19-S45

[21]. Vardar S, Buduneli E, Türkoðlu O, Berdeli AH, Baylas H, Ba ${ }^{\circ}$ kesen A, et al. Therapeutic versus prophylactic plus therapeutic administration of omega-3 fatty acid on endotoxin-induced periodontitis in rats. J Periodontol 2004;75:1640-6

[22]. Sun D, Krishnan A, Zaman K, Lawrence R, Bhattacharya A, Fernandes G. Dietary n-3 fatty acids decrease osteoclastogenesis andloss of bone mass in ovariectomized mice. J Bone Miner Res 2003;18:1206-16

[23]. Kesavalu L, Vasudevan B, Raghu B, Browning E, Dawson D, Novak JM, et al. Omega-3 fatty acid effect on alveolar bone loss in rats. Journal of Dental Research 2006:85:648-52

[24]. Hasturk H, Kantarci A, Ohira T, Arita M, Ebrahimi N, Chiang N, et al. RvE1 protects from local inflammation and osteoclastmediated bone destruction in periodontitis. Faseb Journal.2006; 20:401-3

[25]. Campan P, Planchand PO, Duran D. Pilot study on n-3 polyunsaturated fatty acids in the treatment of human experimental gingivitis. Journal of Clinical Periodontol 1997; 24:907-13

[26]. Eberhard J, Heilmann F, Açil Y, Albers HK, Jepsen S. Local application of n-3 or n-6 polyunsaturated fatty acids in the treatment of human experimental gingivitis. Journal of Clinical Periodontology 2002; 29:364-9

[27]. Rosenstein ED, Kushner LJ, Kramer N, Kazandjian G. Pilot study of dietary fatty acid supplementation in the treatment of adult periodontitis. Prostaglandins Leukot Essent Fatty Acids 2003; 68:213-8

[28]. Naqvi AZ, Buettner C, Phillips RS, Davis RB, Mukamal KJ. n-3 fatty acids and periodontitis in US adults. J Am Diet Assoc 2010; 110:1669-75.

[29]. El-Sharkawy H, Aboelsaad N, Eliwa M, Darweesh M, Alshahat M, Kantarci A, et al. Adjunctive treatment of chronic periodontitis with daily dietary supplementation with Omega-3 fatty acids and low-dose aspirin. JPeriodontol 2010;81:1635-43

[30]. Baillie R., Takada R., Nakamura M., Clarke S. Coordinate induction of peroxisomal acyl-CoA oxidase and UCP-3 by dietary fish oil: A mechanism for decreased body fat deposition. Prostaglandins Leukot. Essent. Fatty Acids. 1999; 60:351-356.

[31]. Belzung F., Raclot T., Groscolas R. Fish oil n-3 fatty acids selectively limit the hypertrophy of abdominal fat depots in growing rats fed high-fat diets. Am. J. Physiol. 1993; 264:R1111-R1118.

[32]. Cunnane S., McAdoo K., Horrobin D. n-3 essential fatty acids decrease weight gain in genetically obese mice. Br. J. Nutr. 1986; 56:87-95.

[33]. Garaulet M., Hernandez-Morante J., Lujan J., Tebar F., Zamora S. Relationship between fat cell size and number and fatty acid composition in adipose tissue from different fat depots in overweight/obese humans. Int. J. Obes. 2006; 30:899-905

[34]. Kabir M., Skurnik G., Naour N., Pechtner V., Meugnier E., Rome S., Quignard-Boulange A., Vidal H., Slama G., Clement K., Guerre-Millo M., Rizkalla S. Treatment for 2 mo with n-3 polyunsaturated fatty acids reduces adiposity and some atherogenic factors but does not improve insulin sensitivity in women with type 2 diabetes: A randomized controlled study. Am. J. Clin. Nutr. 2007; 86:1670-1679.

[35]. Todoric J., Löffler M., Huber J., Bilban M., Reimers M., Kadl A., Zeyda M., Waldhäusl W., Stulnig T. Adipose tissue inflammat ion induced by high-fat diet in obese diabetic mice is prevented by n-3 polyunsaturated fatty acids. Diabetologia. 2006;49:2109-2119.

[36]. Anthony Yates et al. evaluation of lipid profiles and the use of omega - 3 essential fatty acid in professional football players. Sports health 2009; vol 1(1):21-30.

[37]. Goh YK, Jumpsen JA, Ryan EA, Clandinin MT. Effect of omega 3 fatty acid on plasma lipids, cholesterol and lipoprotein fatty acid content in NIDDM patients. Diabetologia. 1997 Jan;40(1):45-52.

[38]. Paraskevas S, Huizinga JD, Loos BG. A systematic review and meta-analyses on C-reactive protein in relation to periodontitis. J Clin Periodontol. 2008; 35:277-290.

[39]. Vardar-Sengul S, Buduneli N, Buduneli E, Kardesler L, Baylas H, Atilla G, et al. Dietary supplementation of omega-3 fatty acid and circulating levels of interleukin-1beta, osteocalcin, and C-reactive protein in rats. J Periodontol. 2006; 77:814-820.

[40]. Mori TA, Woodman RJ, Burke V, Puddey IB, Croft KD, Beilin LJ. Effect of eicosapentaenoic acid and docosahexaenoic acid on oxidative stress and inflammatory markers in treated-hypertensive type 2 diabetic subjects. Free Radic Biol Med. 2003; 35:772781 .

[41]. Madsen T, Christensen JH, Blom M, Schmidt EB. The effect of dietary n-3 fatty acids on serum concentrations of C-reactive protein: a dose-response study. Br J Nutr. 2003; 89:517-522.

[42]. Geelen A, Brouwer IA, Schouten EG, Kluft C, Katan MB, Zock PL. Intake of n-3 fatty acids from fish does not lower serum concentrations of C-reactive protein in healthy subjects. Eur J Clin Nutr. 2004; 58:1440-1442. 
Tables

\begin{tabular}{|l|l|l|l|l|l|}
\hline GROUPS & BASELINE & $\begin{array}{l}\text { AFTER } \\
\text { MONTH }\end{array}$ & $\begin{array}{l}\text { AFTER } \\
\text { MONTH }\end{array}$ & F-VALUE & P-VALUE \\
\hline GROUP A (TEST) & $2.31(0.42)$ & $1.21(0.13)$ & $1.10(0.11)$ & 185.405 & 0.001 \\
\hline $\begin{array}{l}\text { GROUP } \\
\text { CONTROL) }\end{array}$ & $\mathbf{2 . 2 7 ( 0 . 2 8 )}$ & $1.26(0.21)$ & $1.19(0.17)$ & 141.333 & 0.001 \\
\hline
\end{tabular}

TABLE 1: PLAQUE SCORES ACROSS THE GROUPS

\begin{tabular}{|l|l|l|l|l|l|}
\hline GROUPS & BASELINE & $\begin{array}{l}\text { AFTER } \\
\text { MONTH }\end{array}$ & $\begin{array}{l}\text { AFTER } \\
\text { MONTH }\end{array}$ & F-VALUE & P-VALUE \\
\hline TEST GROUP & $2.38(0.31)$ & $1.21(0.09)$ & $1.08(0.16)$ & 281.622 & 0.001 \\
\hline $\begin{array}{l}\text { CONTROL } \\
\text { GROUP }\end{array}$ & $2.31(0.31)$ & $1.18(0.14)$ & $1.05(0.12)$ & 359.745 & 0.001 \\
\hline
\end{tabular}

TABLE 2: GINGIVAL SCORES ACROSS THE GROUPS

\begin{tabular}{|l|l|l|l|l|l|}
\hline GROUPS & BASELINE & $\begin{array}{l}\text { AFTER } \\
\text { MONTH }\end{array}$ & $\begin{array}{l}\text { AFTER } \\
\text { MONTH }\end{array}$ & F-VALUE & P-VALUE \\
\hline TEST GROUP & $3.39(0.28)$ & $1.95(0.27)$ & $1.66(0.40)$ & 275.714 & 0.001 \\
\hline $\begin{array}{l}\text { CONTROL } \\
\text { GROUP }\end{array}$ & $3.35(0.30)$ & $2.31(0.32)$ & $1.86(0.35)$ & 170.199 & 0.001 \\
\hline
\end{tabular}

TABLE 3: BLEEDING SCORES ACROSS THE GROUPS

\begin{tabular}{|l|l|l|l|l|l|}
\hline GROUPS & BASELINE & $\begin{array}{l}\text { AFTER 1 } \\
\text { MONTH }\end{array}$ & $\begin{array}{l}\text { AFTER } \\
\text { MONTH }\end{array}$ & F-VALUE & P-VALUE \\
\hline TEST GROUP & $5.45(0.42)$ & $4.45(0.71)$ & $4.30(0.79)$ & 65.619 & 0.001 \\
\hline $\begin{array}{l}\text { CONTROL } \\
\text { GROUP }\end{array}$ & $5.50(0.53)$ & $4.58(0.80)$ & $4.56(0.80)$ & 79.341 & 0.001 \\
\hline
\end{tabular}

TABLE 4: PROBING POCKET DEPTH ACROSS THE GROUPS

\begin{tabular}{|l|l|l|l|l|l|}
\hline GROUPS & BASELINE & $\begin{array}{l}\text { AFTER } \\
\text { MONTH }\end{array}$ & $\begin{array}{l}\text { AFTER } \\
\text { MONTH }\end{array}$ & F-VALUE & P-VALUE \\
\hline TEST GROUP & $5.55(0.52)$ & $4.59(0.77)$ & $4.56(0.80)$ & 90.846 & 0.001 \\
\hline $\begin{array}{l}\text { CONTROL } \\
\text { GROUP }\end{array}$ & $5.42(0.37)$ & $4.59(0.77)$ & $4.56(0.80)$ & 26.614 & 0.001 \\
\hline
\end{tabular}

TABLE 5: CLINICAL ATTACHMENT LEVEL ACROSS THE GROUPS

\begin{tabular}{|l|l|l|l|l|l|}
\hline GROUPS & BASELINE & $\begin{array}{l}\text { AFTER } \\
\text { MONTH }\end{array}$ & $\begin{array}{l}\text { AFTER } \\
\text { MONTH }\end{array}$ & F-VALUE & P-VALUE \\
\hline TEST GROUP & $236.60(7.09)$ & $235.55(6.19)$ & $230.40(6.21)$ & 23.491 & 0.001 \\
\hline $\begin{array}{l}\text { CONTROL } \\
\text { GROUP }\end{array}$ & $239.10(9.18)$ & $235.45(7.56)$ & $237.50(9.32)$ & 4.008 & 0.036 \\
\hline
\end{tabular}

TABLE 6 : CHOLESTROL LEVELS ACROSS THE GROUPS

\begin{tabular}{|l|l|l|l|l|l|}
\hline GROUPS & BASELINE & $\begin{array}{l}\text { AFTER } \\
\text { MONTH }\end{array}$ & $\begin{array}{l}\text { AFTER } \\
\text { MONTH }\end{array}$ & F-VALUE & P-VALUE \\
\hline TEST GROUP & $\mathbf{3 8 . 2 0}(3.45)$ & $\mathbf{3 9 . 2 0 ( 3 . 3 8 )}$ & $41.70(3.43)$ & 38.196 & 0.001 \\
\hline $\begin{array}{l}\text { CONTROL } \\
\text { GROUP }\end{array}$ & $\mathbf{3 6 . 8 5 ( 3 . 5 2 )}$ & $\mathbf{3 7 . 4 0 ( 4 . 2 3 )}$ & $\mathbf{3 8 . 3 0 ( 3 . 9 2 )}$ & $\mathbf{3 . 6 6 1}$ & $\mathbf{0 . 0 5 4}$ \\
\hline
\end{tabular}

TABLE 7: HDL LEVELS ACROSS THE GROUPS

\begin{tabular}{|l|l|l|l|l|l|}
\hline GROUPS & BASELINE & AFTER 1 MONTH & AFTER 3 MONTH & F-VALUE & P-VALUE \\
\hline TEST GROUP & $162.80(8.85)$ & $162.35(8.61)$ & $158.10(10.18)$ & 17.448 & 0.001 \\
\hline $\begin{array}{l}\text { CONTROL } \\
\text { GROUP }\end{array}$ & $164.55(9.13)$ & $162.55(7.74)$ & $162.15(8.73)$ & 8.384 & 0.001 \\
\hline
\end{tabular}

TABLE 8: LDL LEVELS ACROSS THE GROUPS

\begin{tabular}{|l|l|l|l|l|l|}
\hline GROUPS & BASELINE & $\begin{array}{l}\text { AFTER } \\
\text { MONTH }\end{array}$ & $\begin{array}{l}\text { AFTER } \\
\text { MONTH }\end{array}$ & F-VALUE & P-VALUE \\
\hline TEST GROUP & $221.90(15.29)$ & $220.50(15.25)$ & $214.80(14.42)$ & 33.080 & 0.001 \\
\hline $\begin{array}{l}\text { CONTROL } \\
\text { GROUP }\end{array}$ & $265.35(64.26)$ & $265.10(63.81)$ & $266.75(63.86)$ & 2.332 & 0.135 \\
\hline
\end{tabular}

TABLE 9: TRIGLYCERIDE LEVELS ACROSS THE GROUPS 


\begin{tabular}{|l|l|l|l|l|l|}
\hline GROUPS & BASELINE & $\begin{array}{l}\text { AFTER } \\
\text { MONTH }\end{array}$ & $\begin{array}{l}\text { AFTER } \\
\text { MONTH }\end{array}$ & F-VALUE & P-VALUE \\
\hline TEST GROUP & $9.74(3.62)$ & $8.50(3.57)$ & $7.47(2.68)$ & 14.526 & 0.001 \\
\hline $\begin{array}{l}\text { CONTROL } \\
\text { GROUP }\end{array}$ & $\mathbf{9 . 7 3 ( 3 . 5 2 )}$ & $\mathbf{9 . 0 5 ( 3 . 5 3 )}$ & $\mathbf{8 . 2 0}(2.52)$ & 9.762 & 0.001 \\
\hline
\end{tabular}

TABLE 10: CRP LEVELS ACROSS THE GROUPS

Figures

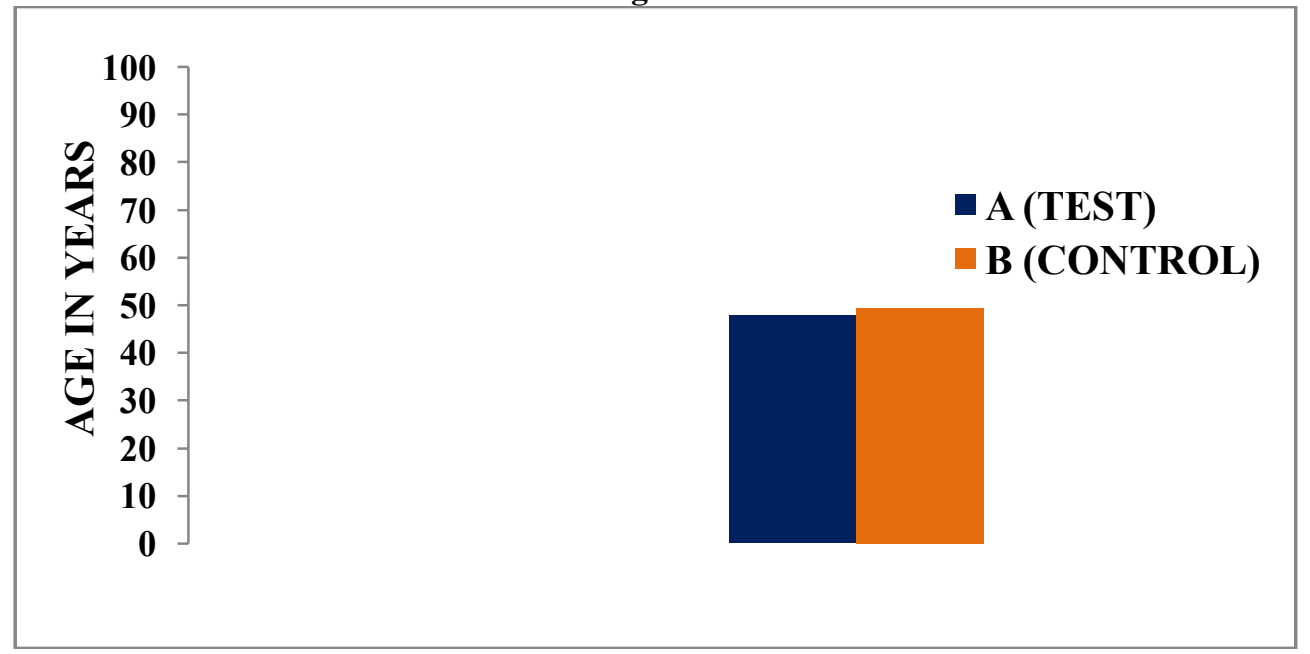

FIGURE 1: AGE DISTRIBUTION AMONG GROUPS

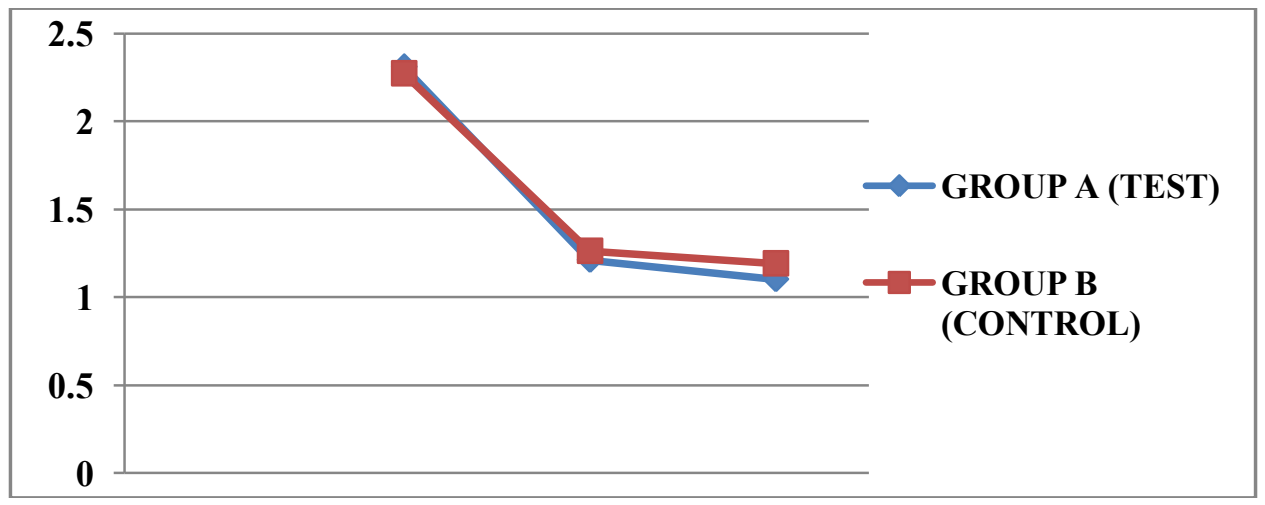

FIGURE 2: PLAQUE SCORES ACROSS THE GROUP

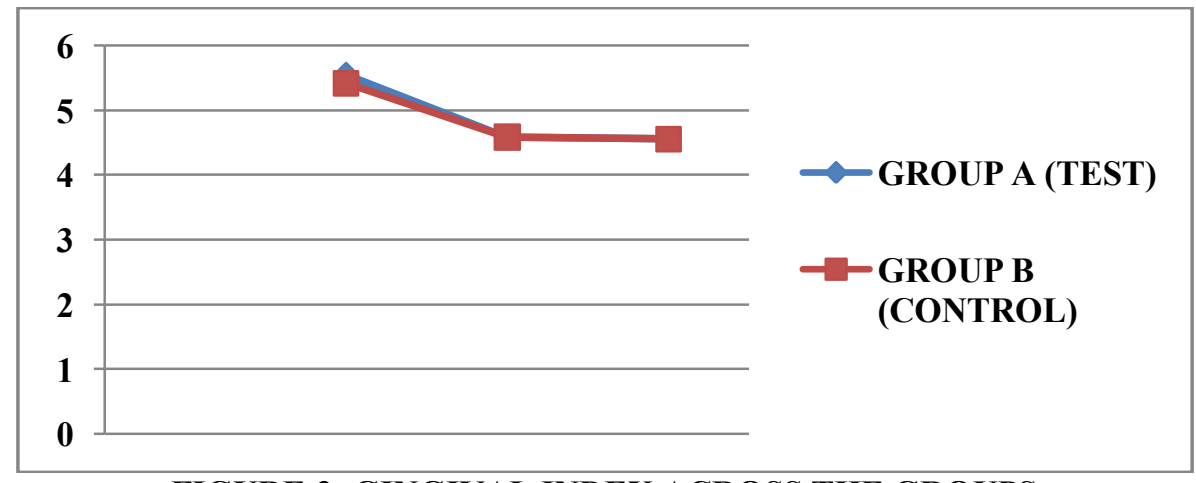

FIGURE 3: GINGIVAL INDEX ACROSS THE GROUPS 


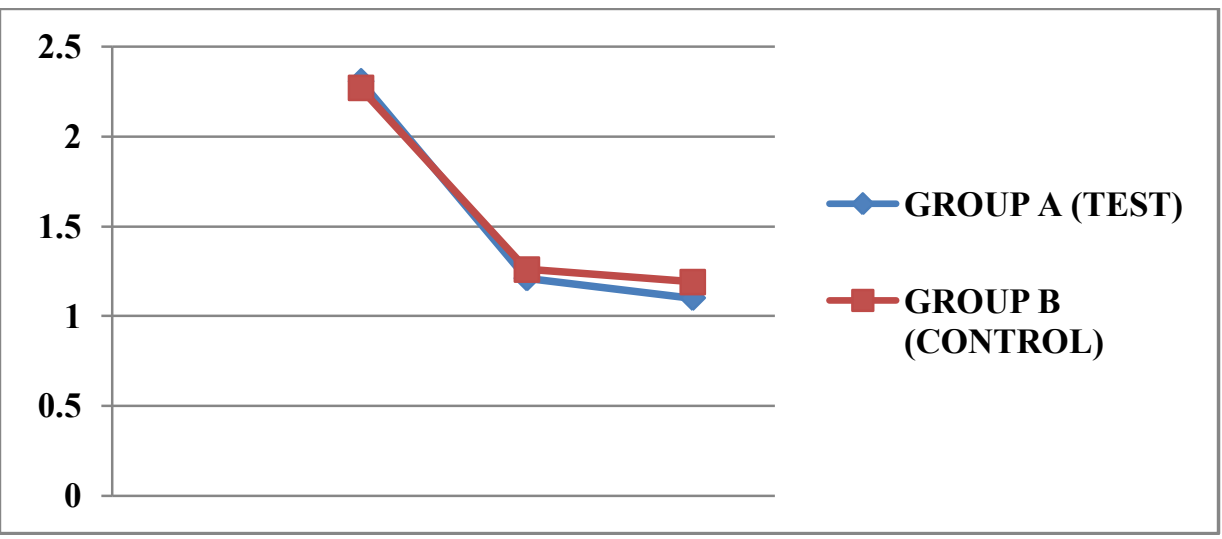

FIGURE 4: BLEEDING SCORES ACROSS THE GROUPS

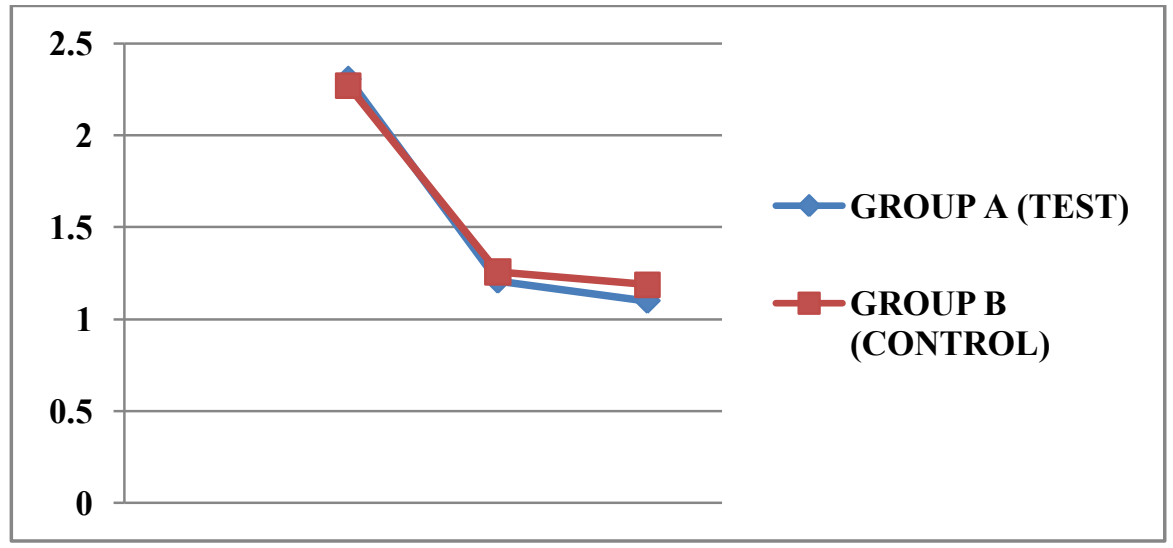

FIGURE 5: PROBING POCKET DEPTH ACROSS THE GROUPS( mm)

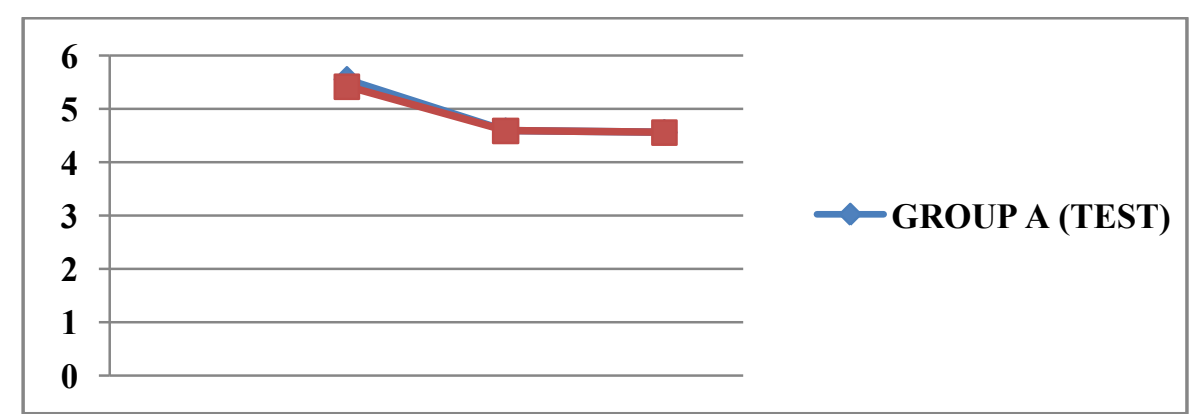

FIGURE 6: CLINICAL ATTACHMENT LEVEL ACROSS THE GROUPS (mm) 


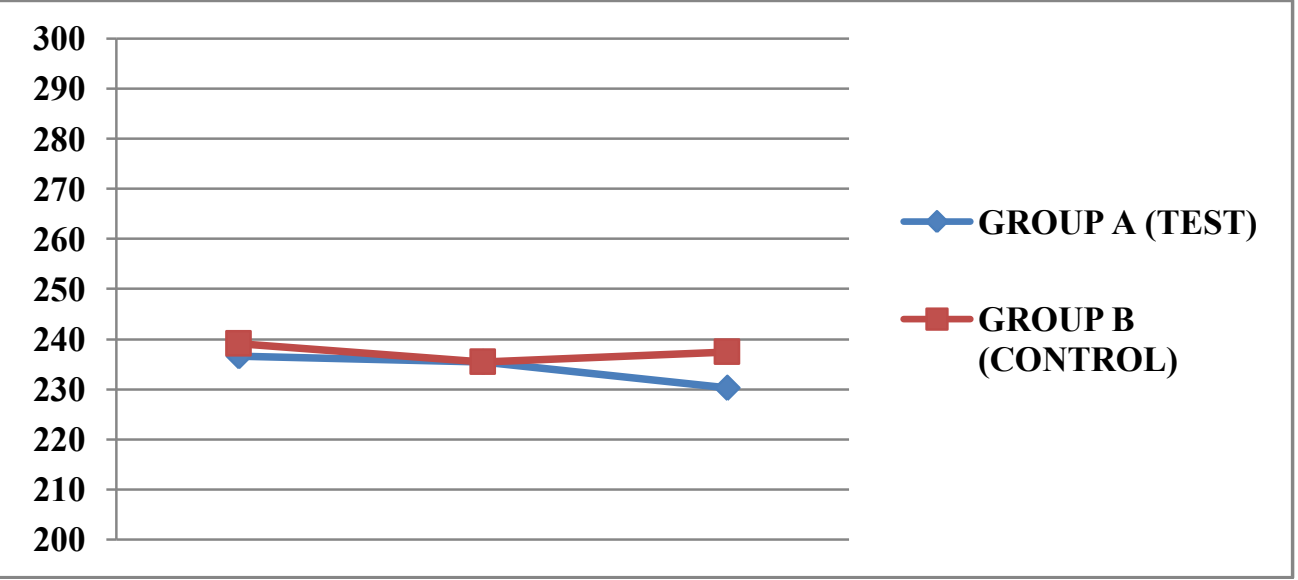

FIGURE 7: CHOLESTROL LEVELS ACROSS THE GROUPS (mg/dl)

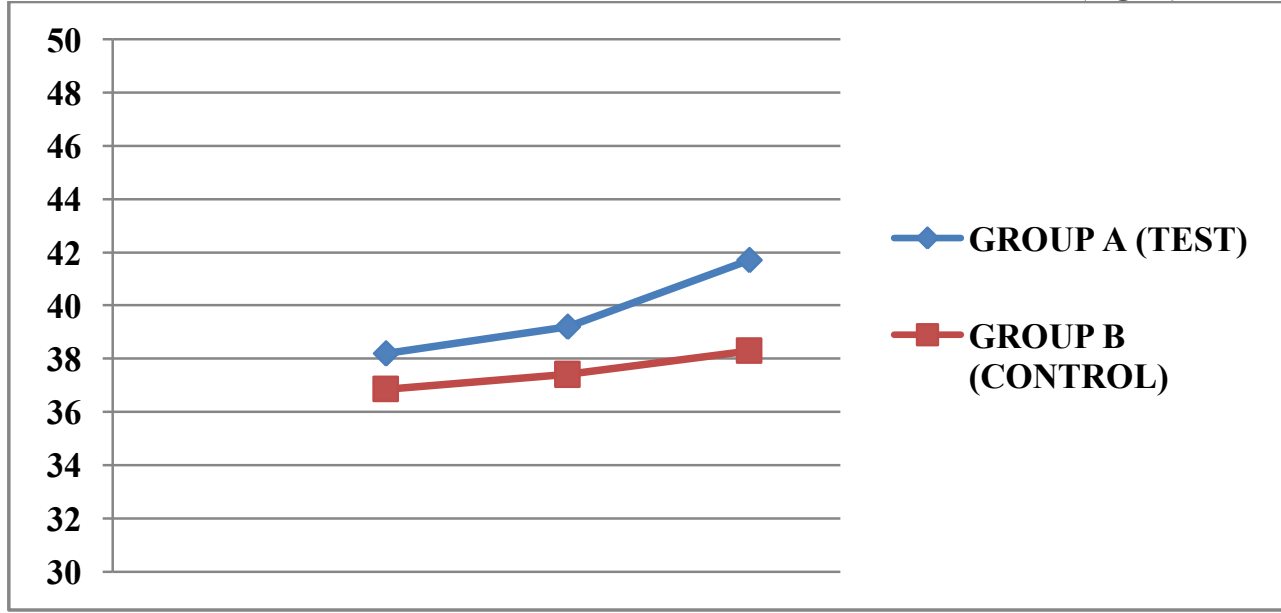

FIGURE 8: HDL LEVELS ACROSS THE GROUPS (mg/dl)

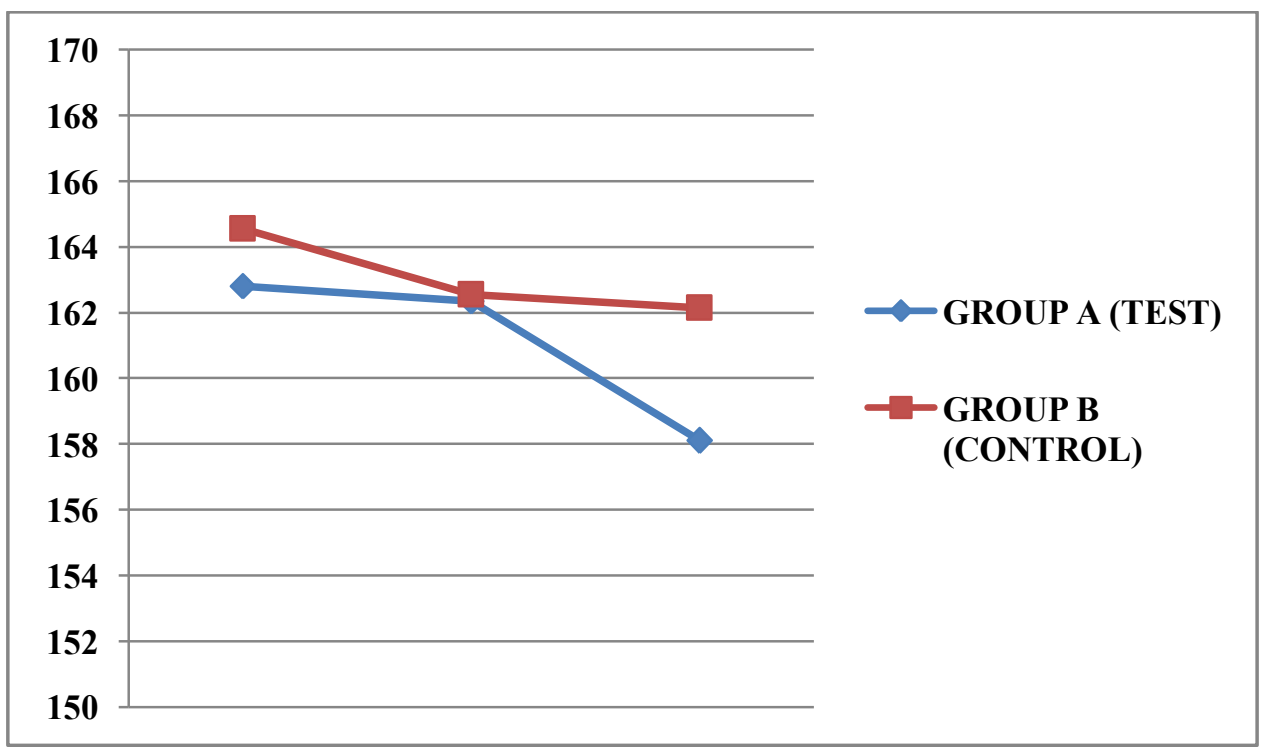

FIGURE 9: LDL LEVELS ACROSS THE GROUPS (mg/dl) 


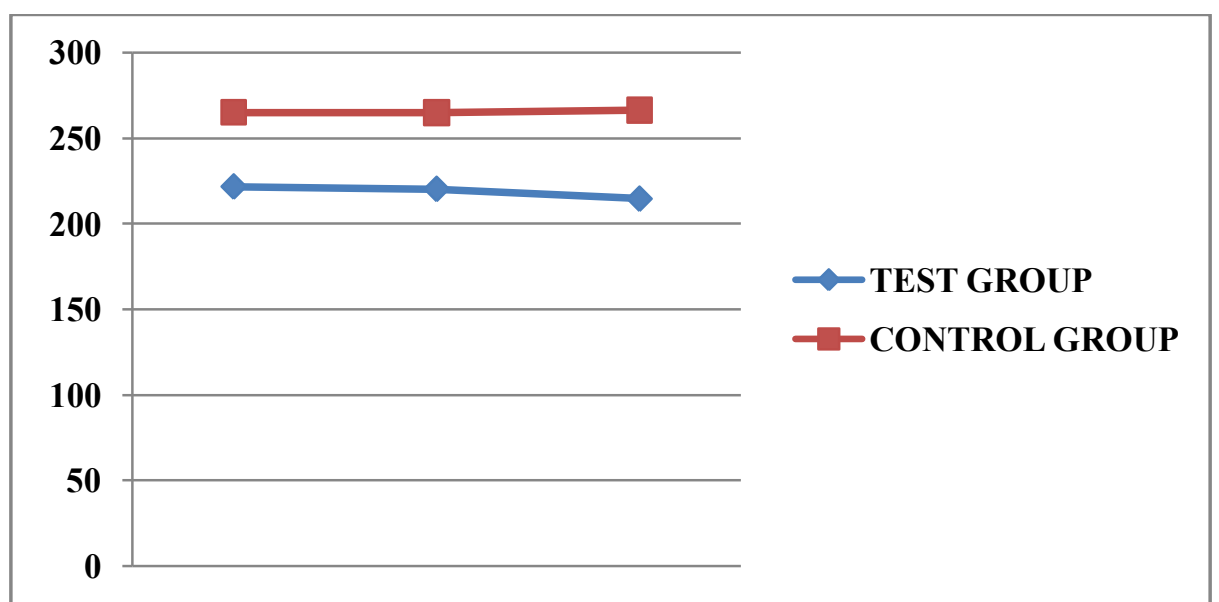

FIGURE 10: TRIGLYCERIDE LEVELS ACROSS THE GROUPS (mg/dl)

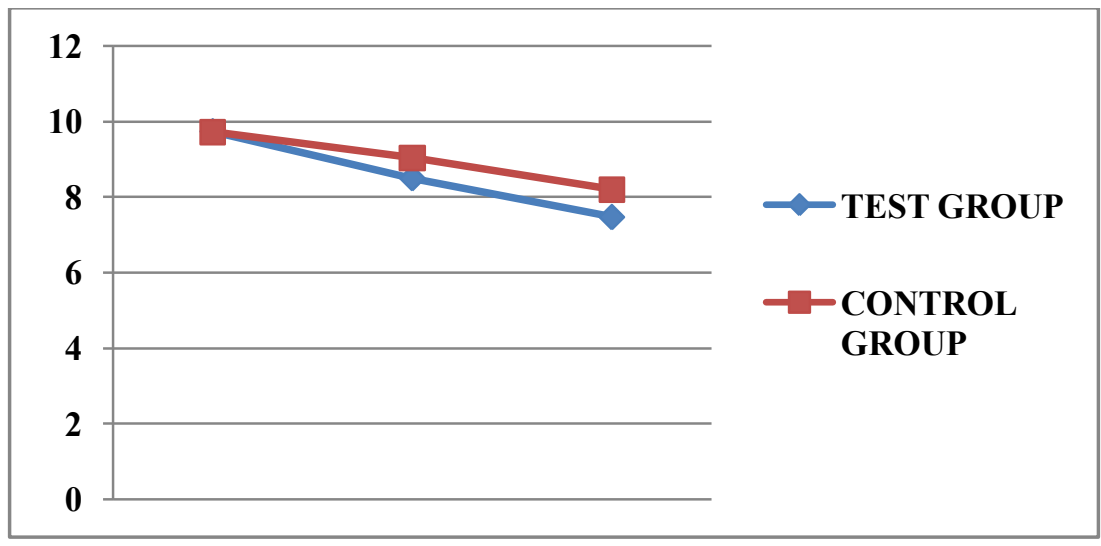

FIGURE 11: CRP LEVELS ACROSS THE GROUPS (mg/dl) 